Le travail de la soie, une voie pour l'exercice de la liberté individuelle des femmes à Tours au XVIII siècle?

\title{
Célia Drouault
}

\section{(2) OpenEdition}

1 Journals

Édition électronique

URL : http://journals.openedition.org/abpo/436

DOI : $10.4000 /$ abpo.436

ISBN : 978-2-7535-1507-9

ISSN : 2108-6443

\section{Éditeur}

Presses universitaires de Rennes

\section{Édition imprimée}

Date de publication : 30 septembre 2007

Pagination : 159-168

ISBN : 978-2-7535-0554-4

ISSN : 0399-0826

\section{Référence électronique}

Célia Drouault, «Le travail de la soie, une voie pour l'exercice de la liberté individuelle des femmes à Tours au XVIII' siècle? ", Annales de Bretagne et des Pays de l'Ouest [En ligne], 114-3| 2007, mis en ligne le 30 septembre 2009, consulté le 19 avril 2019. URL : http://journals.openedition.org/abpo/436 ; DOI $10.4000 / a b p o .436$ 


\title{
Le travail de la soie, une voie pour l'exercice de la liberté individuelle des femmes à Tours au XVIII ${ }^{\mathrm{e}}$ siècle?
}

\author{
Célia Drouault \\ étudiante, \\ CEHVI - université François-Rabelais - Tours
}

Le travail a souvent été considéré comme un des principaux moyens d'émancipation de la femme puisqu'il lui permet, dans certaines conditions, d'accéder à une indépendance économique et financière. Au XVIII siècle, en ville, parmi les métiers organisés en jurandes, la plupart des fonctions interdites aux femmes sont celles situées aux plus hauts niveaux de la hiérarchie professionnelle. Sans doute, quelques activités leur sont-elles exclusivement réservées : les lingères, les couturières, les bouquetières à Paris par exemple, sont organisées en jurandes féminines employant un nombre non négligeable de femmes ${ }^{1}$, qui ont une identité propre. Toutefois, même si certains métiers féminins ont pris un grand essor au XVIII ${ }^{\mathrm{e}}$ siècle, comme celui des marchandes de mode, par exemple, la plupart d'entre eux sont peu reconnus socialement et mal rémunérés.

Les femmes sont présentes dans les activités manufacturières et en particulier dans l'industrie textile : elles sont sollicitées pour la fabrication des tissus, leur transformation, leur entretien, leur réparation... Toutefois, une industrie textile en particulier a retenu notre attention dans le cadre d'une étude sur le travail des femmes : celle de la soie. En effet, la soie est une matière première précieuse, qui présente un intérêt financier important, surtout dans une ville comme Tours où la manufacture de la soie est une

1. La communauté des couturières compte environ une centaine de membres, en 1765, à Tours, mais il est difficile de connaître les chiffres réels (Arch. dép. d'Indre-etLoire, 2B 457, " Rolle d'imposition sur les couturières de Tours » du 26 juin 1765). À Paris, selon Cynthia Truant, les couturières sont plus de 3000 et les lingères environ 2000 (" La maîtrise d'une identité? Corporations féminines à Paris aux XVII et XVIII ${ }^{\mathrm{e}}$ siècles ", dans : Métiers, corporations et syndicalisme, sous la direction de M. ZANCARINI-Fournel, Toulouse, PUM, 1996, p. 55-69). 
mono-industrie. De plus, les villes possédant une telle manufacture sont rares au XVIII ${ }^{\mathrm{e}}$ siècle (Lyon, Nîmes, Paris). Enfin, le métier d'ouvrier en soie est d'autant plus intéressant à étudier qu'il y existe une grande similitude entre l'activité des hommes et celle des femmes : au tissage, ils font le même travail, la répartition des tâches n'est pas toujours sexuée ${ }^{2}$.

\section{La manufacture de soie tourangelle}

Cette " manufacture ", spécifiquement urbaine, est, comme presque toutes les manufactures textiles, dispersée. Elle se présente sous la forme de petits ateliers domestiques, situés dans des maisons particulières et éparpillés dans quelques paroisses ${ }^{3}$. Physiquement, la manufacture est donc peu visible, mais le fait qu'elle soit présente partout, permet à ses membres de se rencontrer dans toute la ville : "Au lieu de quelques manufactures de soie, contenant chacune quelques centaines de métiers groupés dans un espace restreint, c'était comme une cité dans la ville et comme un monde de métiers, dont le bruissement animait du matin au soir les quartiers urbains et suburbains ${ }^{4}$. "L'organisation économique de cette manufacture est très hiérarchisée. Au bas de l'échelle se trouvent la plupart des métiers spécifiquement féminins, qui peuvent aussi être à la campagne : les rouetteuses, les encaneuses, les moulinières, les dévideuses, les ourdisseuses. Ensuite viennent ceux qui travaillent au tissage sur les métiers : les plus nombreux sont les ouvriers[ères] en soie, mais les maîtres-ouvriers y oeuvrent également, pour leur compte ou pour autrui. Dominique Godineau, en se fondant sur différents travaux de recherche, rappelle que, même si ces derniers possèdent une maîtrise et dirigent un atelier, ils sont sous la dépendance économique des marchands-fabricants; leur statut est proche de celui des salariés... Enfin, au sommet de cette hiérarchie se trouvent les marchandsmaîtres-ouvriers, les marchands-fabricants et les négociants qui contrôlent la production et la commercialisation de la soie.

Cette industrie n'est pas originale dans son organisation, puisque toutes les industries textiles sont organisées sur ce modèle, mais elle l'est par la place qu'elle accorde aux femmes, que ce soit dans la sphère privée ou dans la sphère publique. Michelle Perrot remet en question, dès 1984, l'opposition trop simpliste entre la sphère publique, qui serait réservée aux hommes, et la sphère privée, féminine : "La frontière du public et du privé est changeante, sinueuse et traverse même le micro-espace domestique $^{5}$." Cette remarque pose naturellement la question des conséquences

2. Inversement, dans les métiers de l'alimentation, il y a une spécialisation des tâches.

3. Il y a souvent trois métiers par logement : un pour le maître, un pour sa femme et un pour un compagnon.

4. Bossebceuf, Louis, "Histoire de la fabrique de soieries de Tours, des origines au XIX ${ }^{\mathrm{e}}$ siècle ", MSAT, tome 41, L. Pericat, Tours, 1900, p. 222.

5. Perrot, Michelle, "Les femmes, le pouvoir, l'histoire ", Une histoire des femmes estelle possible?, Marseille, Rivages, 1984, p. 216. Elle revient également sur cette question dans Les femmes ou les silences de l'histoire, Paris, Flammarion, 1998, 494 p. 
que le travail peut avoir sur la vie privée, en particulier sur l'émancipation des femmes... Pour aborder ce problème de l'articulation entre sphère privée et sphère publique, ce sont les ouvrières en soie qui ont retenu notre attention, car ce sont des femmes dont l'activité professionnelle peut être étudiée. En effet, il est rare, en général, que les notaires ou les juges précisent la profession exercée par les femmes. Ce silence s'explique par certaines conventions ou convenances sociales tacites, ainsi que par le refus de reconnaître leur activité professionnelle : les femmes de boulangers ne sont pas considérées comme des " travailleuses ", alors qu'elles sont à la boutique, pendant que leur mari est au fournil, par exemple.

La soie nécessite des méthodes de production qui sont plus complexes que le tissage des toiles, par exemple, et impose le recours à une maind'œuvre plus qualifiée, pour laquelle l'apprentissage est plus long et plus coûteux 6 . Au regard de ces difficultés, il aurait été possible de croire que les femmes étaient absentes de ces métiers. Or, selon l'« Etat des déclarations [du nombre des métiers] de la fabrique " de $1783^{7}$, sur les 676 ouvriers employés par les 40 marchands-fabricants de la ville, possédant des métiers, 202 sont des femmes, soit $30 \%$. De plus, en moyenne, de 1753 à 1788 , les femmes représentent $50 \%$ des apprentis et des compagnons ouvriers en soie : 13 apprentisses sont reçues, en moyenne, par an et environ 10 compagnonnes ${ }^{8}$.

Cette nombreuse main-d'œuvre féminine très qualifiée permet une meilleure reconnaissance du travail des femmes : les donneurs d'ouvrage s'accordent aisément à dire que les femmes sont plus soigneuses et plus minutieuses que les hommes. Cette reconnaissance est surtout importante lorsqu'elle passe par la rédaction de règlements concernant leur statut. Ces derniers sont particulièrement nombreux, en particulier en ce qui concerne la situation des filles, des femmes et des veuves de maîtres-ouvriers en soie. Seules les célibataires majeures, qui composent en réalité la majorité des ouvrières en soie, ne font pas l'objet de règlements spécifiques puisqu'elles sont soumises aux mêmes règlements que les hommes. Les ouvrières en soie ont non seulement une place importante dans la principale industrie de la ville, mais cette place, loin d'être ignorée par les pouvoirs publics, fait l'objet de toutes les attentions, surtout lorsqu'un flou juridique existe.

Les femmes occupent parfois dans le domaine professionnel une place tellement importante que le lieutenant général de police doit leur rappeler quelles en sont les limites : « il n'y a qu'un seul état où les femmes puissent être regardées comme des chefs; sçavoir, la Communauté des Lingères à

6. Il faut 5 ans comme apprenti, puis 5 ans comme compagnon, pour pouvoir devenir maître.

7. Arch. dép. d'Indre-et-Loire, E 466, «État des déclarations [du nombre des métiers] de la fabrique ", pour l'année 1783.

8. Arch. dép. d'Indre-et-Loire, E 467, pour le « Registre des apprentis " et E 469, pour le "Registre des Compagnons ". Voir les graphiques 1 et 2 en fin d'article. 
Paris ". La communauté des marchands-fabricants, à Tours comme à Lyon ${ }^{9}$, n'apprécie pas non plus, par exemple, que les « femmes des Ouvriers \& des Compagnons [...] s'arrogent le droit de [leur] demander un Billet, \& en cas de refus de [les] traduire au Bureau, que cette nouveauté blesse toutes les règles ${ }^{10}$ ". Pour les gardes-jurés, les ouvrières tourangelles ont donc atteint et même dépassé en 1750 la limite supportable de leur " émancipation professionnelle " et la décision est prise, par le procureur du roi, d'interdire aux femmes d'ouvriers de travailler pour un autre maître que celui de leur mari. Cette décision a finalement pour conséquence de renvoyer les femmes auprès de leur mari, dans un espace de la sphère privée ${ }^{11}$. Néanmoins, l'application de cette règle est de courte durée puisque dès 1754, le Sieur Reverdy, Lieutenant Général de police, traite du cas où « lesdits MaîtresOuvriers \& leurs Femmes seulement, demeurans \& vivans ensemble, travailleront pour différents Maîtres ${ }^{12}$ ". Cette situation est très proche de celle existant à Lyon où, théoriquement, le travail féminin est interdit à l'extérieur, et ce jusqu'en 1786, mais où cette loi est en pratique peu appliquée... Ces ordonnances montrent à quel point les femmes ont leur place au sein de la cité et peuvent être au centre de différentes préoccupations, juridiques et sociales. Il existe une forme de paradoxe entre la présence nécessaire des femmes dans cette industrie et les efforts désespérés des pouvoirs publics et des autorités de la jurande, pour les cantonner dans la sphère domestique. La participation des femmes à cette manufacture leur permet de sortir de la sphère privée, relevant du droit familial, pour entrer dans la sphère du travail salarié, encadré par une jurande. Elles y sont en contact direct avec l'extérieur (leur patron, les autres ouvriers...), même si elles travaillent en fait dans des ateliers d'autant plus familiaux qu'ils sont situés dans le logement familial...

L'importance prise par le travail des femmes au sein d'une société peut également se mesurer en fonction de leur accès aux rangs les plus élevés de l'industrie. Pour le monde de la soie, ce sont, en ordre croissant, les marchands maîtres-ouvriers en soie, les marchands-fabricants et les négociants qui figurent au sommet de l'échelle; or, les femmes ne sont pas exclues de ces fonctions supérieures. Par exemple, la veuve Viot-Baudichon est la sixième fabricante en soie de la ville, en nombre de métiers ${ }^{13}$, mais ce sont surtout les pourcentages qui témoignent de la présence féminine : en 1765, $14,5 \%$ des marchands fabricants sont des femmes, et $23 \%$ en $1783^{14}$. Les

9. Les marchands fabricants de Lyon obtiennent en 1744 qu'il soit interdit aux épouses de se salarier.

10. Arch. dép. d'Indre-et-Loire, C 104, extrait de l'" Ordonnance du Lieutenant Général de Police des 17 et 23 janvier 1750 contre les ouvriers, leurs femmes \& Enfans, Compagnons et Pillage des Desseins".

11. Puisque le domicile est souvent un atelier et que la femme n'a plus à sortir de chez elle pour aller travailler.

12. Arch. dép. d'Indre-et-Loire, C 104, Ordonnance du Lieutenant de police du 4 mars 1754

13. Elle en a plus de 50 (Arch. dép. d'Indre-et-Loire, E 466, "État des déclarations [du nombre de métiers] de la Fabrique ", 1783).

14. Cette augmentation peut être le fait de la réforme de 1776. 
femmes peuvent avoir des responsabilités élevées au sein du monde de la soie, même si les plus favorisées restent, sans conteste, les filles et les veuves de maîtres-ouvriers en soie ou de marchands-fabricants ${ }^{15}$. L'accès à ce niveau de la hiérarchie professionnelle permet aux femmes de jouir de grandes responsabilités, du même statut légal, et surtout social, que les hommes.

En revanche, les femmes sont absentes des niveaux plus " politiques" : elles ne peuvent, par exemple, être gardes-jurés qu'au sein des corporations féminines. Or, pour Cynthia Truant, " sans cette possibilité d'accès au pouvoir de la hiérarchie officielle qui dirige une corporation, contrôle ses finances et règle le travail, les femmes, même si elles forment une minorité importante, ont peu de poids ${ }^{16}$ ". Cette absence n'est donc pas une particularité tourangelle.

\section{Les femmes dans la manufacture}

Les ouvrières en soie présentent différents traits communs ${ }^{17}$ : environ $50 \%$ de ces femmes ont entre 20 et 27 ans, elles demeurent majoritairement dans les paroisses de Notre-Dame-la-Riche à l'ouest ${ }^{18}$, de Saint-Pierre-duBoile au centre, et de Saint Etienne en périphérie méridionale de la ville ${ }^{19}$. $84 \%$ des ouvrières en soie sont des femmes jeunes et célibataires, pour qui le travail est un moyen de subsistance, mais également d'émancipation. Il est habituel de dire que le sort des jeunes femmes célibataires n'est pas toujours enviable sous l'Ancien Régime, que la plupart d'entre elles n'ont le choix qu'entre le mariage et le couvent. Elles vivent presque toujours sous la puissance d'un homme : leur père quand elles sont jeunes, leur mari lorsqu'elles sont mariées. Or, lorsque les célibataires majeures trouvent un emploi assez bien rémunéré ${ }^{20}$, elles n'ont plus à faire ce choix : elles peuvent enfin profiter de leurs différents droits, même si la pression sociale est, quant à elle, toujours présente. Le fait que les femmes célibatai-

15. Voir par exemple, "Statuts, Ordonnances et Reglements pour le Corps et la Communauté des Marchands Maîtres Ouvriers en Draps d'or, d'argent et de soye de la Ville et Faubourg de Tours donnez et concedez par sa Majesté en 1667 " (Arch. dép. d'Indre-et-Loire, E 461) ainsi que le " Projet des Statuts et Règlements concernants le commerce, art, et manufacture de draps d'or, d'argent et de soye, et autres mélangées de soye, laine, fils et cotton qui se font dans la Ville Faubourgs et Banlieux de Touraine" (Arch. dép. d'Indre-et-Loire, E 466).

16. Truant, Cynthia, "La maîtrise d'une identité... ", art. cit., dans Métiers, corporations et syndicalisme, sous la direction de M. ZANCARINI-FouRnEL, Toulouse, PUM, 1996, p. 55-69.

17. Ces informations ont été relevées sur un échantillon de 147 ouvrières en soie.

18. Cette paroisse, située à l'ouest de la ville, est la plus vaste et la plus peuplée de la ville. Les métiers de la soie représentent $61 \%$ de l'activité professionnelle de la paroisse, alors qu'ils ne représentent que $25,5 \%$ pour la ville entière.

19. La présence féminine dans ces paroisses est respectivement de $46,5 \%, 9,5 \%$ et $9,5 \%$, soit $65 \%$.

20. Les ouvrières employées au tirage de la soie gagnent généralement, à la journée de travail, de 1 livre à 1 livre 10 sols (Arch. dép. d'Indre-et-Loire, C 107, « État de la dépense faite au tirage des soies » du 22 juin au 23 juillet 1750). 
res, en particulier les jeunes, aient accès à un certain nombre de métiers, relativement bien payés, doit jouer en faveur de leur liberté personnelle : ce sont les premières à avoir véritablement le choix entre la sphère privée et la sphère publique. Elles n'ont pas à s'occuper des enfants, ce qui leur laisse plus de temps pour s'engager dans différentes formes de vie sociale et religieuse.

La liberté financière donnée aux femmes par le travail a également rendu possible des comportements particuliers. En raison de la concentration des ateliers dans des paroisses bien définies, les ouvrières en soie se regroupent géographiquement et pratiquent largement la cohabitation. Celle-ci n'a pas seulement lieu entre membres de la même famille ${ }^{21}$, elle peut également se pratiquer entre « collègues " de travail. L'étude des " donations par forme de droit d'accroissement " est, de ce point de vue, particulièrement riche d'enseignements ${ }^{22}$. Ces donations, émanant de femmes célibataires, sont une forme de solidarité, d'assistance mutuelle, rendue possible par l'établissement d'une communauté formelle de biens ${ }^{23}$, en particulier quand arrive la vieillesse ${ }^{24}$. Sur 18 donations, concernant 36 femmes, 5 émanent de femmes travaillant dans le monde de la soie, soit $28 \%$ : trois paires de dévideuses, une d'ouvrière en soie, une moulinière avec une ourdisseuse. Ce comportement témoigne d'une évolution importante, surtout à la fin du siècle ${ }^{25}$ : les femmes célibataires ne souhaitent pas demeurer seules, mais elles ne sont pas pour autant déterminées à vivre avec un homme. Le travail, en particulier celui de la soie, a permis aux femmes de s'émanciper de la protection, financière et sociale, des hommes. Cynthia Truant a également repéré " quelques cas de filles majeures qui se sont mises en ménage avec d'autres femmes [...], pour un temps assez long et elles ont reproduit ainsi quelques-unes des fonctions d'un mariage hétérosexuel ". Les attitudes les plus " libérales" se retrouvent en fait dans ce milieu, ainsi que dans celui de la domesticité, et accessoirement dans celui de la prostitution, car les femmes célibataires y sont souvent très nombreuses. Il est très difficile de recueillir des renseignements sur le comportement " privé " des ouvrières en soie, mais ces cohabitations laissent supposer que d'autres formes de sociabilités féminines existent.

Le $\mathrm{XVIII}^{\mathrm{e}}$ siècle a vu se développer l'émancipation professionnelle des femmes, que ce soit par rapport à leur mari, puisqu'elles ne travaillent plus nécessairement pour eux ou avec eux ou à travers la place qui leur est réservée dans la société, puisqu'elles ne sont plus cantonnées aux métiers dits "naturels", et ce même dans une ville comme Tours. Le travail offre

21. Entre parents et enfants, et plus fréquemment entre sœurs.

22. Ces donations se font, comme les autres, devant notaires, et sont soumises, depuis 1731, à l'Insinuation.

23. L'article CCXXXI de la coutume de Touraine autorise indirectement la communauté de biens entre individus non unis par les liens du mariage.

24. Les comparaisons sont nombreuses avec le mariage tardif.

25. Dans la documentation utilisée, seulement 4 demandes ont été relevées dans la première partie du siècle, contre 14 dans les années 1780 . 
aux femmes, qu'elles soient célibataires, mariées ou veuves, des moyens d'émancipation sociale et d'indépendance, elles sont reconnues en tant qu'unités productives à part entière, avec leurs spécificités et leurs difficultés. Pour Simone Roux, grâce au travail, « les femmes ne sont pas obligatoirement dans la dépendance matérielle de leur père ou de leur mari, [...] elles peuvent avoir des libertés d'action, [...] elles peuvent trouver dans le travail rémunéré non seulement des ressources mais aussi une dignité, un rang, une reconnaissance de leur rôle dans le monde où elles vivent ${ }^{26}$ ". Cette réflexion peut s'appliquer à la situation des ouvrières en soie tourangelles du XVIII ${ }^{\mathrm{e}}$ siècle : le travail féminin ne permet pas seulement aux femmes d'entrer dans la sphère publique, il modifie également la manière dont elles perçoivent le domaine privé et domestique.

Le travail de la soie a apporté un certain nombre de moyens de liberté aux femmes ${ }^{27}$. Pour les jurandes féminines, cette constatation s'impose, puisque les femmes occupent tous les postes à responsabilité. En revanche, le métier d'ouvrier en soie n'est pas exclusivement masculin, il est véritablement mixte, et le pouvoir exercé par les femmes dans le monde de la soie a donc nécessairement plus de poids. Cette implication des femmes peut être considérée comme une valorisation du travail des femmes, du moins dans ce domaine, mais il est tout aussi possible qu'il soit le signe d'une dévalorisation du métier. Quoi qu'il en soit, l'activité féminine a indéniablement permis aux femmes des comportements qui leur étaient interdits auparavant. Le célibat féminin n'a jamais été favorisé par les pouvoirs publics, pourtant, la forte présence des célibataires dans ces métiers est la preuve de son importance. Les femmes exercent ainsi effectivement les droits que le Droit leur attribue ${ }^{28}$, mais elles le font même évoluer en leur faveur : jamais la Coutume de Touraine, ni celle de Paris d'ailleurs, n'avait prévu explicitement l'existence des donations par forme de droit d'accroissement. La pratique a non seulement fait apparaître ce droit, mais elle l'a également fait accepter par la société entière.

26. Roux, Simone, "Les femmes dans les métiers parisiens : XIII- $-\mathrm{xv}^{\mathrm{e}}$ siècle ", dans : Métiers, corporations et syndicalisme, sous la direction de M. ZANCARINI-FOURNEL, Toulouse, PUM, 1996, p. 14.

27. Il faudrait, par exemple, se demander si le travail des femmes permet à la famille une existence plus facile, s'il a changé les rapports entre mari et femme.

28. Le Droit est constitué de la Coutume, de la Loi royale, des règlements de métier. 
Annexe 1 - La place des femmes parmi les apprentis ouvriers en soie à Tours, de 1754 à 1785

(Ces graphiques permettent de comprendre à quel point la condition professionnelle des femmes doit être prise en compte : en moyenne, plus de $50 \%$ des apprentis et des compagnons ouvriers en soie sont des femmes.)

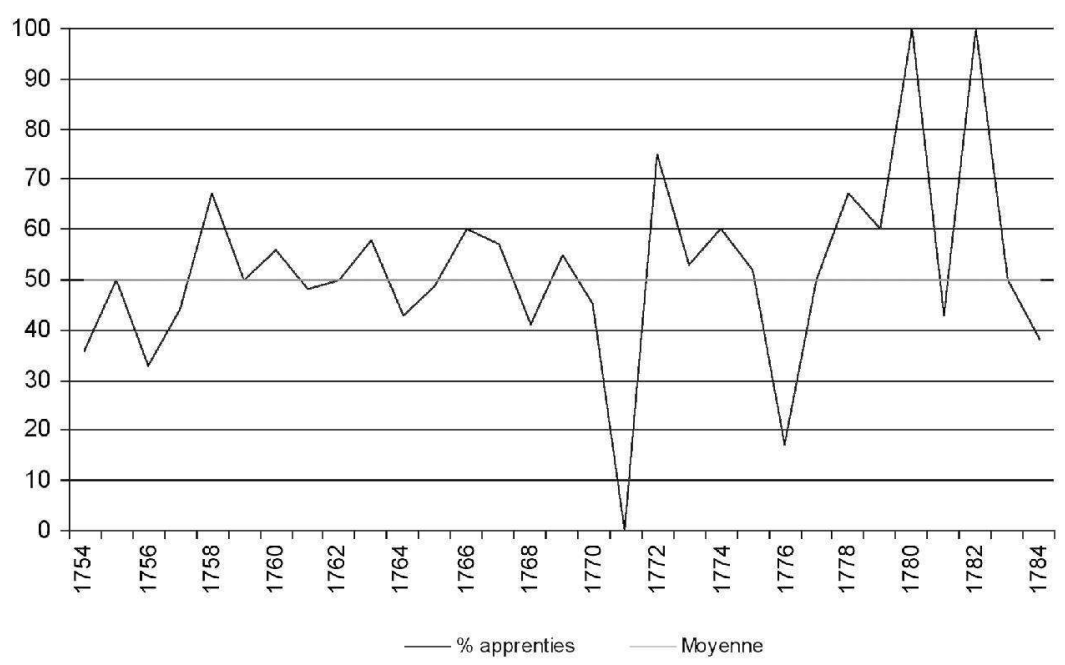

Annexe 2 - La place des femmes parmi les compagnons ouvriers en soie à Tours, de 1754 à 1785

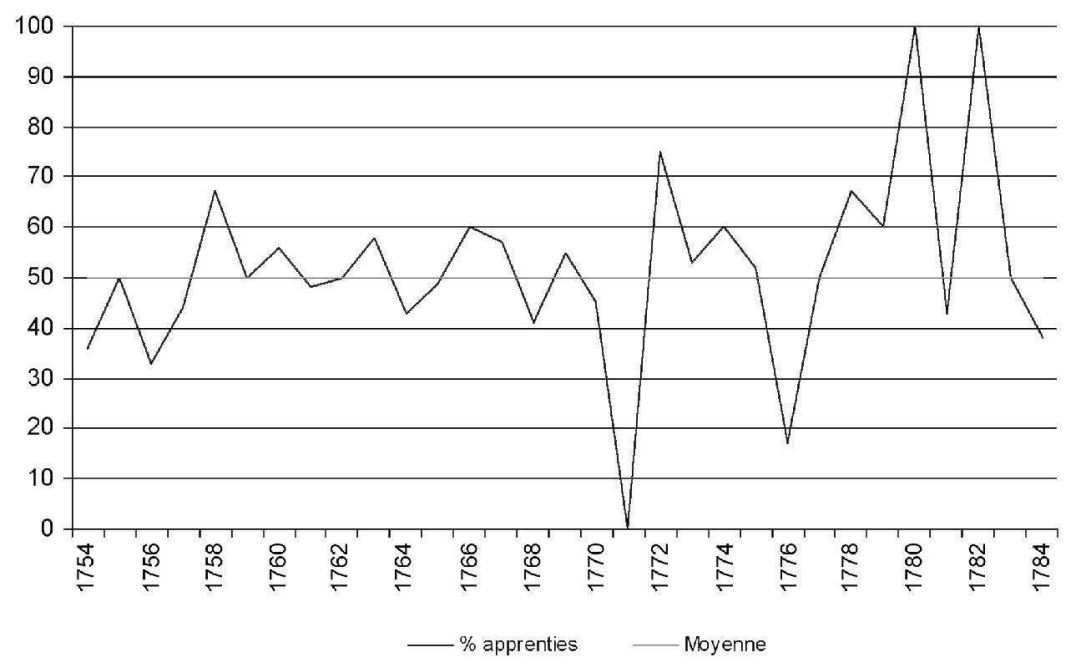




\section{Bibliographie}

Bossebceuf, Louis, "Histoire de la fabrique de soieries de Tours, des origines au XIX ${ }^{\mathrm{e}}$ siècle ", MSAT, tome 41, L. Pericat, Tours, 1900, p. 193-528.

Budin, Jean-François, "Les ouvrières de la soie à Lyon au XVIII ${ }^{\mathrm{e}}$ siècle ", $127^{\mathrm{e}}$ congrès des sociétés historiques et scientifiques, Nancy, avril 2002.

GARDEN, Maurice, Lyon et les Lyonnais au XVIII siècle, Paris, Flammarion, 1984, $773 \mathrm{p}$.

GODART, Justin, L'Ouvrier en soie, monographie du tisseur lyonnais, étude historique, économique et sociale. $1^{\text {re }}$ partie : « La réglementation du travail, le maître ouvrier en draps d'or, d'argent et de soie, de l'établissement de la manufacture à Lyon (1466) au décret des 2-17 mars 1791 portant suppression de toutes les maîtrises et jurandes ", Thèse, Lyon, imprimerie de E. Nicolas, 1899, 543 p.

Godineau, Dominique, Les Femmes dans la société française (XVI'-XVII siècle), Paris, Armand Colin, 2003, $252 \mathrm{p}$.

HAFTER, Daryl M., European women and preindustrial craft, Bloomington, Indianapolis, Indiana University Press, 1995, 204 p.

JORDI, Emmanuelle, Les contrats d'apprentissage à Tours. L'exemple des apprentis ouvriers en soie dans la seconde moitié du XVIII siècle, mémoire de maîtrise, sous la direction de Brigitte Maillard, Tours, 1999.

MAILlARD, Brigitte, "Un lent assoupissement, XVII - -XVIII e siècle ", dans CHEVALIER, Bernard, Histoire de Tours, Toulouse, Privat, 1985, p. 179-220.

PERROT, Michelle, Les Femmes ou les silences de l'Histoire, Paris, Flammarion, 1998.

Roux, Simone, "Les femmes dans les métiers parisiens : XIII $-\mathrm{XV}^{\mathrm{e}}$ siècle ", dans ZANCARINI-FOURNEL, Michelle (dir.), Métiers, corporations et syndicalisme, Toulouse, PUM, 1996, p. 13-30.

SchmitT, Marie-Thérèse, La paroisse de Notre-Dame-la-Riche à Tours sous l'Ancien Régime, mémoire de maîtrise sous la direction de Robert Sauzet, Université de Tours, 1979, $101 \mathrm{p}$.

TRuANT, Cynthia, «La maîtrise d'une identité? Corporations féminines à Paris aux $\mathrm{XVII}^{\mathrm{e}}$ et XVIII" siècles ", sous la direction de ZANCARINI-FouRnEL Michelle, Toulouse, PUM, 1996, p. 55-69. 


\section{RÉSUMÉ}

Dans la ville de Tours, le travail de la soie est quasiment une mono-industrie; les femmes, et en particulier les ouvrières en soie, peuvent y obtenir, sinon une indépendance, du moins une certaine émancipation. Les preuves de cette émancipation peuvent être de trois ordres : la place tenue par ces femmes aussi bien dans la sphère privée, les ouvrières travaillant souvent à leur domicile, que dans la sphère publique; le fait que cette émancipation soit au centre des préoccupations juridiques et sociales; et enfin les traits propres à ces femmes. Pour la plupart elles ont en effet entre vingt et vingt-sept ans et elles sont en majorité célibataires. Enfin, cette émancipation, notamment des femmes seules, favorisée ou encouragée par le travail de la soie, se manifeste par un changement dans la manière dont elles perçoivent le domaine privé et domestique, par leur tendance à développer des formes de solidarité et de sociabilité féminines, qui en retour permettent à ces femmes de garder une forme d'indépendance. Le travail de la soie peut donc bien être considéré comme une voie pour l'exercice de la liberté individuelle des femmes tourangelles au XVIII ${ }^{\mathrm{e}}$ siècle.

\section{ABSTRACT}

In the city of Tours, silk work constituted practically the sole local industry. It was through silk that women, and in particular those who worked in the production of the raw material, were able to achieve, if not independence, at least a certain form of emancipation. Three factors can be put forward as evidence of this newfound emancipation: the role of women both in the private domain, since the workers very often worked in their own homes, and in the public sphere; the fact that this emancipation was to be found at the heart of legal and social preoccupations of the time; and last of all, the very characteristics of these women: most of them were aged between twenty and twenty-seven, and single for the most part. Last of all, this emancipation, in particular of single women, aided or even encouraged by the silk-working industry, was manifested by a change in the way they perceived the private and domestic domain and by their tendency to develop new forms of feminine solidarity and sociability, which in turn enabled these women to maintain this form of independence they had achieved. Silk working can therefore be considered as one of the main means by which the women of Tours in the 18th century were able to exercise their individual liberty. 